\title{
Autoinflammatory Keratinization Diseases (AiKDs): Expansion of Disorders to Be Included
}

\author{
Masashi Akiyama* \\ Department of Dermatology, Nagoya University Graduate School of Medicine, Nagoya, Japan
}

Keywords: hidradenitis suppurativa, keratosis lichenoides chronica, pityriasis rubra pilaris, porokeratosis, pustular psoriasis

Inflammation caused by the hyper-activation of innate immunity due to genetic factors occasionally leads to inflammatory keratinization diseases of the skin. Such inflammatory keratinization diseases with genetic autoinflammatory pathomechanisms are called "autoinflammatory keratinization diseases" (AiKDs) (1). AiKDs also include disorders with combined pathological mechanisms of autoimmunity and autoinflammation. AiKDs possess primary genetic causal features associated with autoinflammation mainly in the epidermis and in the superficial dermis. Autoinflammation in these regions results in hyperkeratosis in the skin, leading to other cutaneous inflammatory symptoms of AiKDs $(1,2)$. The clinical phenotypes of AiKDs are variable, and each disease has unique characteristic manifestations, although common clinical features are hyperkeratotic lesions with inflammation (Table 1). Most AiKD patients have recurrent and persistent cutaneous lesions.

Initially, AiKDs encompassed generalized pustular psoriasis (GPP), pityriasis rubra pilaris (PRP), and familial keratosis lichenoides chronica $(\mathrm{KLC})(1,2)$. It remains controversial as to whether GPP should be considered an AiKD. Many patients with GPP, acrodermatitis continua, or impetigo herpetiformis have been reported to have IL36RN mutations (3-9). GPP shows massive neutrophil infiltration leading to pustulosis and occasionally erosive skin. The level of hyperkeratosis is variable, depending on the timing, lesions and cases. However, keratinocyte differentiation and proliferation are thought to be frequently affected in the lesional epidermis of GPP, and GPP is considered to be a disease in which keratinization is affected, although hyperkeratosis is not apparent in some cases. In this context, it is reasonable to consider GPP a keratinization disease. In some GPP patients, rare mutations of both IL36RN and CARD14 have been identified (8). This observation indicates that GPP is not caused by monogenic factors of either CARD14 or IL36RN, but that these two genes are quite strong risk factors for GPP onset. Together with the strong HLA-class II associations with GPP, GPP seems to be a polygenic disease, with strong effects from IL36RN mutations. Mouse model results also support this view: Il36rn-null mice never show the disease spontaneously, but need imiquimod as a potential trigger to show GPP-like phenotypes (10). IL36RN mutations are occasionally found in GPP patients with psoriasis, although GPP patients without psoriasis seem to have $\operatorname{IL} 36 R N$ mutations more frequently $(4,9)$. In addition, Arakawa et al. (11) reported that induced IL36RN levels are lower in both GPP patients with and without IL36RN mutations, and proposed "IL36RN insufficiency" in GPP. Consistently, GPP patients can be successfully treated by IL36 signal blockade whether or not IL36RN mutations are present (12). Overall, it seems that the majority of GPP patients have hyperactivation in IL36 signaling. On the other hand, the evidence for autoimmunity in GPP is quite strong (13). Antigen-specific $\mathrm{CD}^{+}{ }^{+} \mathrm{T}$ cell activation has been detected for certain HLA-class II alleles (11). In fact, treatments targeting T-cells are effective against GPP. However, in our initial definition of AiKDs, we defined the concept of AiKDs as encompassing diseases with mixed pathomechanisms of autoinflammation and autoimmunity (1). In cases of GPP, autoinflammation plays a significant role, although autoimmunity is also important. Thus, GPP is thought to be included in AiKDs.

Furthermore, both the CARD14 pathway and the IL36RN pathway are associated not only with GPP, but also with psoriasis vulgaris. GPP and psoriasis vulgaris are thought to belong 
TABLE 1 | Inflammatory keratinization diseases classified as AiKDs to date [adopted and modified from Akiyama et al. (1, 2)].

\begin{tabular}{|c|c|c|}
\hline Disease & $\begin{array}{l}\text { Genetic causative or predisposing factor } \\
\text { (frequency) }\end{array}$ & $\begin{array}{l}\text { Pathogenic mechanism/pathway of inflammation in } \\
\text { keratinocytes }\end{array}$ \\
\hline \multicolumn{3}{|l|}{ Pustular psoriasis and related disorders } \\
\hline \multirow[t]{2}{*}{ Generalized pustular psoriasis (GPP) } & $\begin{array}{l}\text { IL36RN mutations (predominant in early-onset } \\
\text { cases and/or cases without psoriasis vulgaris) }\end{array}$ & $\begin{array}{l}\mathrm{IL} 36 \mathrm{Ra} \downarrow \rightarrow \mathrm{IL}-36 \uparrow \rightarrow \mathrm{MyD} 88 \uparrow \rightarrow \mathrm{NFKB} / \mathrm{MAPK} \uparrow \rightarrow \mathrm{TNF}, \mathrm{IL}-1, \mathrm{IL}-8, \\
\mathrm{IL}-17, \mathrm{IL}-36, \mathrm{CXCL} 1, \mathrm{CXCL2}, \mathrm{CCL} 20 \uparrow\end{array}$ \\
\hline & CARD14 variants (occasional) & $\mathrm{CARD} 14 \uparrow \rightarrow \mathrm{NFKB} \uparrow \rightarrow \mathrm{IL}-36, \mathrm{IL}-8, \mathrm{CXCL} 1, \mathrm{CXCL} 2, \mathrm{CCL} 20 \uparrow$ \\
\hline acrodermatitis continua & IL36RN mutations (occasional) & \\
\hline $\begin{array}{l}\text { palmoplantar pustular psoriasis } \\
\text { (palmoplantar pustulosis) }\end{array}$ & CARD14 variants (occasional) & $\mathrm{CARD} 14 \uparrow \rightarrow \mathrm{NFKB} \uparrow \rightarrow \mathrm{IL}-36, \mathrm{IL}-8, \mathrm{CXCL} 1, \mathrm{CXCL} 2, \mathrm{CCL} 20 \uparrow$ \\
\hline \multicolumn{3}{|l|}{ Pityriasis rubra pilaris (PRP) } \\
\hline PRP type $V$ & CARD14 mutations (predominant) & $\mathrm{CARD} 14 \uparrow \rightarrow \mathrm{NFKB} \uparrow \rightarrow \mathrm{IL}-36, \mathrm{IL}-8, \mathrm{CXCL} 1, \mathrm{CXCL2}, \mathrm{CCL} 20 \uparrow$ \\
\hline Hidradenitis suppurativa (HS) & $\begin{array}{l}\text { mutations in } \gamma \text {-secretase genes, NCSTN, } \\
\text { PSENEN and PSEN1 (infrequent) }\end{array}$ & $\begin{array}{l}\text { Hypothesis: } \gamma \text {-secretase } \downarrow \rightarrow \text { Notch signal } \downarrow \rightarrow \text { aberrant differentiation } \\
\text { and proliferation of the epidermis and the hair follicle epithelium }\end{array}$ \\
\hline porokeratosis & $\begin{array}{l}\text { mutations in mevalonate pathway-related } \\
\text { genes, MVK, MVD, PMVK and FDPS } \\
\text { (predominant) }\end{array}$ & $\begin{array}{l}\text { Hypothesis: MVK, MVD, PMVK, FDPS } \downarrow \rightarrow \text { geranylgeranyl } \\
\text { diphosphate } \downarrow \rightarrow \text { isoprenylated proteins (RAS, lamin B, etc.) } \\
\downarrow \rightarrow \text { aberrant differentiation and proliferation of epidermal keratinocytes }\end{array}$ \\
\hline
\end{tabular}

to the same disease entity on a spectrum. Indeed, CARD14 mutations are associated with both psoriasis vulgaris and GPP, with the strongest CARD14 activity causing GPP $(14,15)$. Psoriasis genome-wide association studies show CARD14 to be a risk gene for psoriasis (16), meaning that a CARD14mediated pathway could be working in psoriasis patients without CARD14 mutations. On the other hand, antigenspecific $\mathrm{CD}^{+} \mathrm{T}$ cell activation occurs in both psoriasis vulgaris and GPP (11). Together with identified autoantigens that activate $\mathrm{CD}^{+} \mathrm{T}$ cells in psoriasis $(17,18)$, this $\mathrm{CD}^{+} \mathrm{T}$ cell activation may represent a common psoriasis pathway that is also observed in GPP. Thus, psoriasis vulgaris also has both autoinflammatory and autoimmune pathogeneses, so it might well be included in AiKDs, because according to its initial definition, AiKDs encompass diseases with mixed pathomechanisms of autoinflammation and autoimmunity (1).

Most PRP type V patients harbor CARD14 mutations (19) and are categorized as having an AiKD, and a small number of PRP cases of other types have CARD14 variants and are also included in AiKDs. Craiglow et al. (20) proposed the term "CARD14-associated papulosquamous eruption" to indicate psoriasis patients and PRP type $\mathrm{V}$ patients carrying CARD14 mutations, and they suggested that psoriasis/PRP type $\mathrm{V}$ patients with CARD14 mutations have characteristic features of early onset, predominant facial skin symptoms, and good response to ustekinumab treatment. Such psoriasis/PRP type V patients with CARD14 mutations are considered to be typical AiKD cases. In addition, some cases with pustular psoriasis may be related to mutations in $A P 1 S 3$ coding adaptor-related protein complex 1, sigma-3 subunit (AP1S3) (21). The AP1S3 molecule is involved in autophagosome formation, especially in keratinocytes. Loss of function of AP1S3 is considered to result in defective autophagy and abnormal accumulation of p62. p62 is known to mediate NFKB activation. Thus, p62 accumulation is thought to lead to NFאB hyper-activation, up-regulation of IL1 signaling, and overexpression of IL-36 (21). In this context, pustular psoriasis associated with AP1S3-including cases with GPP, acrodermatitis continua, and palmoplantar pustulosis-is recognized as an AikD.

Recently, it has been proposed that hidradenitis suppurativa (HS), especially familial cases, be included in AiKDs. Mutations in the $\gamma$-secretase complex genes have been identified in patients and families with HS (22). HS patients carrying mutations in NCSTN, PSENEN, and PSEN1 coding the $\gamma$-secretase complex show hyperkeratotic hair follicle epithelia in the infundibular region, resulting in the occlusion of hair follicles. Such occlusion is considered to be an essential part of the pathogenic mechanism behind HS (22). The plugging of the epidermis by keratinous materials is also a distinctive finding in skin lesions of PRP, a typical AiKD (19). Furthermore, it has been reported that tissue levels of TNF, caspase-1, IL-1, and IL-17 are high in the lesional skin of HS patients $(23,24)$. It might be reasonable to consider that HS, at least HS with $\gamma$-secretase gene mutations, is a clinical entity included in AiKDs because the primary step in the pathogenesis of HS is thought to be hyperkeratosis of the follicular epithelium in the infundibular 
region due to the aberrant activation of innate immunity. Indeed, occasionally, $\mathrm{HS}$ is seen in patients with autoinflammatory disorders including pyoderma gangrenosum and pyogenic sterile arthritis as autoinflammatory syndromes, such as pyoderma gangrenosum, acne and pyogenic arthritis (PAPA) syndrome, pyoderma gangrenosum, acne and HS (PASH) syndrome, and Majeed syndrome (25-29). HS and these autoinflammatory syndromes with HS might share the pathogenic mechanisms of hyperactivated innate immunity resulting in up-regulated production of IL-1 family cytokines and neutrophilic infiltration in the skin $(26,27)$. In considering the pathogenesis of HS, it is meaningful to recognize $\mathrm{HS}$ as an AiKD initiated by the hyperactivation of innate immunity in the epithelium of the follicular infundibulum. Heterozygous mutations in one of the three genes (NCSTN, PSENEN, and PSEN1) encoding the $\gamma$ secretase complex have been reported in HS (22). $\gamma$-secretase is a membrane-bound aspartyl protease complex which works in the intramembrane proteolysis of various membrane proteins, including Notch (28). Notch is a membrane receptor, and the binding of ligands to the extracellular portion of Notch triggers cleavage of the intracellular portion of Notch by $\gamma$-secretase, with the cleaved intracellular domain of Notch moving into the nucleus and regulating expression of various genes (29). In the skin, the differentiation and proliferation of epidermal cells, and the differentiation and maintenance of the epithelium of hair follicles and sebaceous glands are regulated by Notch signaling (22). Thus, reduced enzyme activity of $\gamma$-secretase complex due to mutations in NCSTN, PSENEN, and PSEN1 is considered to down-regulate Notch signaling, resulting in aberrant differentiation and proliferation of the epidermis and the hair follicle epithelium in HS. The malfunction of $\gamma$-secretase was revealed to result in the abnormal differentiation of the epithelium in hair follicles via down-regulation of Notch signals in studies using model mice (22).

Porokeratosis encompasses diverse hyperkeratotic lesions consisting of single or multiple atrophic macules or plaques demarcated by hyperkeratotic ridges. Vertical columns of parakeratotic cells called "cornoid lamellae" are histological findings characteristic of porokeratosis (30). Porokeratosis is obviously a keratinization disease and also is regarded as an inflammatory disorder of the skin (30). In fact, significant inflammation is seen in eruptive pruritic papular porokeratosis, and cutaneous inflammatory disorders including localized cutaneous amyloidosis are complications of porokeratosis cases (30). We have proposed that porokeratosis be categorized as an AiKD. In 2012, mutations in $M V K$, one of the mevalonate pathway genes, were detected as a cause of disseminated superficial actinic porokeratosis (31). To date, four mevalonate pathway genes ( $M V K, M V D, P M V K$, and FDPS) have been identified as causative of porokeratosis (30). The mevalonate pathway is known to produce isoprenoid precursors (30). Isoprenoids are precursors of various important molecules, such as heme A, cholesterol, and isoprenylated proteins (30). Isoprenylated proteins, including RAS and lamin B, regulate the growth and differentiation of cells (30). It was demonstrated that the deficiency of geranylgeranylpyrophosphate, a nonsterol isoprenoid in mevalonate pathway products, causes the activation of inflammasomes via the abnormal function of Rac1 (32). We speculate that malfunctions in the mevalonate pathway might result in the abnormal growth and differentiation of epidermal keratinocytes and autoinflammation in the lesions of porokeratosis. In this context, porokeratosis would be an AiKD, and it is occasionally hereditary (30). Indeed, one of the porokeratosis-causative mevalonate pathway genes, $M V K$, is also causative of hyperimmunoglobulinemia $\mathrm{D}$ and periodic fever syndrome, an established autoinflammatory syndrome (33). Biallelic mutations in $M V K$ cause the more severe, systemic disease, hyperimmunoglobulinemia $\mathrm{D}$ and periodic fever syndrome, and heterozygous mutations in $M V K$ lead to porokeratosis (31).

Porokeratosis patients with causative mevalonate pathway gene mutations have the mutations as heterozygous germline mutations. In skin lesions of porokeratosis, the expression of mutant alleles is higher than that of wild-type alleles, and the predominant expression of mutant alleles is considered to be a trigger for the formation of porokeratotic lesions (30). As a causative mechanism of the predominant expression of mutant alleles and the loss of function of wild-type alleles in porokeratotic lesions, an unelucidated DNA-methylationindependent epigenetic process is presumed in most cases (30), and in addition, genomic recombination which results in homozygosity of mutant alleles has been reported in other cases (30). Aberrant RNA editing was also reported in the lesional tissue of one porokeratosis case (30).

Concerning treatments for AiKDs, based on the autoinflammatory pathogenic mechanisms mentioned above, therapeutic strategies targeting molecules working in autoinflammatory cascades of pathomechanisms in AiKDs, such as cytokines, their receptors, and signal molecules, are expected to bring promising innovative therapies for various diseases included in AiKDs. Indeed, various treatments against pathogenic mechanisms are sufficiently effective for a number of AiKDs.

Successful treatments with granulocyte and monocyte adsorption apheresis (34), anakinra (an IL-1 receptor antagonist) (35), canakinumab (a monoclonal anti-IL-1 $\beta$ antibody) (36), infliximab (a monoclonal anti-human TNF- $\alpha$ antibody) (37), adalimumab (a monoclonal anti-TNF- $\alpha$ antibody) (38), ustekinumab (a monoclonal anti-human IL-12/IL-23 p40 antibody) (39), secukinumab (an anti-IL-17 monoclonal antibody) (40), and ixekizumab (a monoclonal anti-IL17A antibody) (41) have been reported for GPP as an AiKD. Ustekinumab is also sufficiently effective against PRP with CARD14 mutations (42). Concerning treatments for AiKDs due to NLRP1 mutations, the IL-1 receptor antagonist anakinra was reported to be effective in cases of NLRP1-associated autoinflammation with arthritis and dyskeratosis (43). As for treatments for HS, adalimumab, infliximab, anakinra, and ustekinumab have been recommended (23).

The concept of AiKDs has ushered in a new era in inflammatory keratinization disorders. We assume that, in the near future, a growing number of inflammatory keratinization diseases may be recognized to be AiKDs, along with an advanced knowledge of novel pathomechanisms 
working in inflammatory keratinization disorders. The recognition of innate causative/predisposing issues and the precise assessment of their roles in disease etiology from the perspective of AiKDs promise to bring innovations that will afford more precise, targeted, causal therapies for various AiKDs.

\section{AUTHOR CONTRIBUTIONS}

The author confirms being the sole contributor of this work and has approved it for publication.

\section{REFERENCES}

1. Akiyama M, Takeichi T, McGrath JA, Sugiura K. Autoinflammatory keratinization diseases. J Allergy Clin Immunol. (2017) 140:1545-7. doi: 10.1016/j.jaci.2017.05.019

2. Akiyama M, Takeichi T, McGrath JA, Sugiura K. Autoinflammatory keratinization diseases: an emerging concept encompassing various inflammatory keratinization disorders of the skin. J Dermatol Sci. (2018) 90:105-11. doi: 10.1016/j.jdermsci.2018.01.012

3. Marrakchi S, Guigue P, Renshaw BR, Puel A, Pei XY, Fraitag S, et al. Interleukin-36-receptor antagonist deficiency and generalized pustular psoriasis. N Engl J Med. (2011) 365:620-8. doi: 10.1056/NEJMoa1013068

4. Sugiura K, Takemoto A, Yamaguchi M, Takahashi H, Shoda Y, Mitsuma $\mathrm{T}$, et al. The majority of generalized pustular psoriasis without psoriasis vulgaris is caused by deficiency of interleukin-36 receptor antagonist. J Invest Dermatol. (2013) 133:2514-21. doi: 10.1038/jid.2013.230

5. Setta-Kaffetzi N, Navarini AA, Patel VM, Pullabhatla V, Pink AE, Choon SE, et al. Rare pathogenic variants in IL36RN underlie a spectrum of psoriasis-associated pustular phenotypes. J Invest Dermatol. (2013) 133:13669. doi: 10.1038/jid.2012.490

6. Abbas O, Itani S, Ghosn S, Kibbi AG, Fidawi G, Farooq M, et al. Acrodermatitis continua of Hallopeau is a clinical phenotype of DITRA: evidence that it is a variant of pustular psoriasis. Dermatology. (2013) 226:2831. doi: $10.1159 / 000346572$

7. Sugiura K, Oiso N, Iinuma S, Matsuda H, Minami-Hori M, Ishida-Yamamoto A, et al. IL36RN mutations underlie impetigo herpetiformis. J Invest Dermatol. (2014) 134:2472-4. doi: 10.1038/jid.2014.177

8. Mössner R, Wilsmann-Theis D, Oji V, Gkogkolou P, Löhr S, Schulz P, et al. The genetic basis for most patients with pustular skin disease remains elusive. Br J Dermatol. (2018) 178:740-8. doi: 10.1111/bjd.15867

9. Twelves S, Mostafa A, Dand N, Burri E, Farkas K, Wilson R, et al. Clinical and genetic differences between pustular psoriasis subtypes. J Allergy Clin Immunol. (2019) 143:1021-6. doi: 10.1016/j.jaci.2018.06.038

10. Shibata A, Sugiura K, Furuta Y, Mukumoto Y, Kaminuma O, Akiyama M. Tolllike receptor 4 antagonist TAK-242 inhibits autoinflammatory symptoms in DITRA. J Autoimmun. (2017) 80:28-38. doi: 10.1016/j.jaut.2017.01.007

11. Arakawa A, Vollmer S, Besgen P, Galinski A, Summer B, Kawakami Y, et al. Unopposed IL-36 activity promotes clonal CD4+_T-cell responses with IL17A production in generalized pustular psoriasis. J Invest Dermatol. (2018) 138:1338-47. doi: 10.1016/j.jid.2017.12.024

12. Bachelez H, Choon SE, Marrakchi S, Burden AD, Tsai TF, Morita A, et al. Inhibition of the interleukin-36 pathway for the treatment of generalized pustular psoriasis. N Engl J Med. (2019) 380:981-3. doi: 10.1056/ NEJMc1811317

13. Navarini AA, Smith CH, Barker JN, Capon F. Reply to the letter "Earlyonset generalized pustular psoriasis is representative of autoinflammatory keratinization diseases". J Allergy Clin Immunol. (2019) 143:810-1. doi: $10.1016 /$ j.jaci.2018.11.010

14. Jordan CT, Cao L, Roberson ED, Pierson KC, Yang CF, Joyce CE, et al. PSORS2 is due to mutations in CARD14. Am J Hum Genet. (2012) 90:784-95. doi: 10.1016/j.ajhg.2012.03.012

\section{FUNDING}

MA was supported by funding from the Advanced Research and Development Programs for Medical Innovation (AMEDCREST) 19gm0910002h0105 of the Japan Agency for Medical Research and Development (AMED), by Grant-in-Aid for Scientific Research (B) 18H02832 from the Japan Society for the Promotion of Science (JSPS), by a grant from the Ministry of Health, Labor and Welfare of Japan (Heath and Labor Sciences Research Grants; Research on Intractable Disease: H29-029) and by The Uehara Memorial Foundation.

15. Jordan CT, Cao L, Roberson ED, Duan S, Helms CA, Nair RP, et al. Rare and common variants in CARD14, encoding an epidermal regulator of NF-кB, in psoriasis. Am J Hum Genet. (2012) 90:796-808. doi: 10.1016/ j.ajhg.2012.03.013

16. Tsoi LC, Spain SL, Knight J, Ellinghaus E, Stuart PE, Capon F, et al. Identification of 15 new_psoriasis_susceptibility loci highlights the role of innate immunity. Nat Genet. (2012) 44:1341-8. doi: 10.1038/ng.2467

17. Lande R, Botti E, Jandus C, Dojcinovic D, Fanelli G, Conrad C, et al. The antimicrobial peptide LL37 is a T-cell autoantigen in psoriasis. Nat Commun. (2014) 5:5621. doi: 10.1038/ncomms6621

18. Arakawa A, Siewert K, Stöhr J, Besgen P, Kim SM, Rühl G, et al. Melanocyte antigen triggers autoimmunity in human psoriasis. J Exp Med. (2015) 212:2203-12. doi: 10.1084/jem.20151093

19. Takeichi T, Sugiura K, Nomura T, Sakamoto T, Ogawa Y, Oiso N, et al. Pityriasis rubra pilaris type $\mathrm{V}$ as an autoinflammatory disease by CARD14 mutations. JAMA Dermatol. (2017) 153:66-70. doi: 10.1001/ jamadermatol.2016.3601

20. Craiglow BG, Boyden LM, Hu R, Virtanen M, Su J, Rodriguez G, et al. CARD14-associated papulosquamous eruption: a spectrum including features of psoriasis and pityriasis rubra pilaris. J Am Acad Dermatol. (2018) 79:48794. doi: 10.1016/j.jaad.2018.02.034

21. Mahil SK, Twelves S, Farkas K, Setta-Kaffetzi N, Burden AD, Gach JE, et al. AP1S3 mutations cause skin autoinflammation by disrupting keratinocyte autophagy and up-regulating IL-36 production. J Invest Dermatol. (2016) 136:2251-9. doi: 10.1016/j.jid.2016.06.618

22. Pink AE, Simpson MA, Desai N, Trembath RC, Barker JNW. $\gamma$-Secretase mutations in hidradenitis suppurativa: new insights into disease pathogenesis. J Invest Dermatol. (2013) 133:601-7. doi: 10.1038/jid.2012.372

23. Saunte DML, Jemec GBE. Hidradenitis suppurativa: advances in diagnosis and treatment. JAMA. (2017) 318:2019-32. doi: 10.1001/jama.2017.16691

24. Moran B, Sweeney CM, Hughes R, Malara A, Kirthi S, Tobin AM, et al. Hidradenitis suppurativa is characterized by dysregulation of the Th17:Treg cell axis, which is corrected by anti-TNF therapy. J Invest Dermatol. (2017) 137:2389-95. doi: 10.1016/j.jid.2017.05.033

25. Gasparic J, Theut Riis P, Jemec GB. Recognizing syndromic hidradenitis suppurativa: a review of the literature. J Eur Acad Dermatol Venereol. (2017) 31:1809-16. doi: 10.1111/jdv.14464

26. Vinkel C, Thomsen SF. Autoinflammatory syndromes associated with hidradenitis suppurativa and/or acne. Int J Dermatol. (2017) 56:811-8. doi: $10.1111 /$ ijd.13603

27. De Vita V, McGonagle D. Hidradenitis suppurativa as an autoinflammatory keratinization disease. J Allergy Clin Immunol. (2018) 141:1953. doi: 10.1016/ j.jaci.2018.01.010

28. Li T, Wen H, Brayton C, Das P, Smithson LA, Fauq A, et al. Epidermal growth factor receptor and notch pathways participate in the tumor suppressor function of $\gamma$-secretase. J Biol Chem. (2007) 282:32264-73. doi: 10.1074/ jbc.M703649200

29. Oswald F, Täuber B, Dobner T, Bourteele S, Kostezka U, Adler G, et al. p300 acts as a transcriptional coactivator for mammalian Notch1. Mol Cell Biol. (2001) 21:7761-74. doi: 10.1128/MCB.21.22.7761-777 4.2001 
30. Zhang Z, Li C, Wu F, Ma R, Luan J, Yang F, et al. Genomic variations of the mevalonate pathway in porokeratosis. Elife. (2015) 4:e06322. doi: 10.7554/eLife.06322.021

31. Zhang SQ, Jiang T, Li M, Zhang X, Ren YQ, Wei SC, et al. Exome sequencing identifies MVK mutations in disseminated superficial actinic porokeratosis. Nat Genet. (2012) 44:1156-60. doi: 10.1038/ng.2409

32. van der Burgh R, Ter Haar NM, Boes ML, Frenkel J. Mevalonate kinase deficiency, a metabolic autoinflammatory disease. Clin Immunol. (2013) 147:197-206. doi: 10.1016/j.clim.2012.09.011

33. Houten SM, Kuis W, Duran M, de Koning TJ, van Royen-Kerkhof A, Romeijn GJ, et al. Mutations in MVK, encoding mevalonate kinase, cause hyperimmunoglobulinaemia D and periodic fever syndrome. Nat Genet. (1999) 22:175-7. doi: 10.1038/9691

34. Sugiura K, Haruna K, Suga Y, Akiyama M. Generalized pustular psoriasis caused by deficiency of interleukin-36 receptor antagonist successfully treated with granulocyte and monocyte adsorption apheresis. J Eur Acad Dermatol Venereol. (2014) 28:1835-6. doi: 10.1111/jdv.12383

35. Hüffmeier U, Wätzold M, Mohr J, Schön MP, Mössner R. Successful therapy with anakinra in a patient with generalized pustular psoriasis carrying IL36RN mutations. Br J Dermatol. (2014) 170:202-4. doi: 10.1111/bjd.12548

36. Gómez-García F, Sanz-Cabanillas JL, Viguera-Guerra I, Isla-Tejera B, Nieto AV, Ruano J. Scoping review on use of drugs targeting interleukin 1 pathway in DIRA and DITRA. Dermatol Ther. (2018) 8:539-56. doi: $10.1007 /$ s13555-018-0269-7

37. Sugiura K, Endo K, Akasaka T, Akiyama M. Successful treatment with infliximab of sibling cases with generalized pustular psoriasis caused by deficiency of interleukin-36 receptor antagonist. J Eur Acad Dermatol Venereol. (2015) 29:2054-6. doi: 10.1111/jdv.12590

38. Hospach T, Glowatzki F, Blankenburg F, Conzelmann D, Stirnkorb C, Müllerschön CS, et al. Scoping review of biological treatment of deficiency of interleukin-36 receptor antagonist (DITRA) in children and adolescents. Pediatr Rheumatol Online J. (2019) 17:37. doi: 10.1186/s12969-019-0338-1
39. Arakawa A, Ruzicka T, Prinz JC. Therapeutic efficacy of interleukin 12/interleukin 23 blockade in generalized pustular psoriasis regardless of IL36RN mutation status. JAMA Dermatol. (2016) 152:825-8. doi: 10.1001/ jamadermatol.2016.0751

40. Cordoro KM, Ucmak D, Hitraya-Low M, Rosenblum MD, Liao W. Response to interleukin (IL)-17 inhibition in an adolescent with severe manifestations of IL-36 receptor antagonist deficiency (DITRA). JAMA Dermatol. (2017) 153:106-8. doi: 10.1001/jamadermatol.2016.3490

41. Nagata M, Kamata M, Fukaya S, Hayashi K, Fukuyasu A, Tanaka T, et al. Realworld single-center experience with 10 cases of generalized pustular psoriasis successfully treated with ixekizumab. J Am Acad Dermatol. (2019) 82:758-61. doi: 10.1016/j.jaad.2019.09.040

42. Eytan O, Sarig O, Sprecher E, van Steensel MA. Clinical response to ustekinumab in familial pityriasis rubra pilaris caused by a novel mutation in CARD14. Br J Dermatol. (2014) 171:420-2. doi: 10.1111/bjd. 12952

43. Grandemange S, Sanchez E, Louis-Plence P, Tran Mau-Them F, Bessis D, Coubes C, et al. A new autoinflammatory and autoimmune syndrome associated with NLRP1 mutations: NAIAD (NLRP1-associated autoinflammation with arthritis and dyskeratosis). Ann Rheum Dis. (2017) 76:1191-8. doi: 10.1136/annrheumdis-2016-210021

Conflict of Interest: The author declares that the research was conducted in the absence of any commercial or financial relationships that could be construed as a potential conflict of interest.

Copyright (C) 2020 Akiyama. This is an open-access article distributed under the terms of the Creative Commons Attribution License (CC BY). The use, distribution or reproduction in other forums is permitted, provided the original author(s) and the copyright owner(s) are credited and that the original publication in this journal is cited, in accordance with accepted academic practice. No use, distribution or reproduction is permitted which does not comply with these terms. 